\title{
COPD patients hospitalized with exacerbations have greater cognitive impairment than patients hospitalized with decompensated heart failure
}

This article was published in the following Dove Press journal:

Clinical Interventions in Aging

\author{
Mohani-Preet K Bajaj \\ Daniel R Burrage 2 \\ Andrew Tappouni ${ }^{3}$ \\ James W Dodd ${ }^{4}$ \\ Paul W Jones ${ }^{2}$ \\ Emma H Baker ${ }^{2}$
}

'Neurosciences Research Centre, Molecular and Clinical Sciences Research Institute, St George's University of London, London, UK; ${ }^{2}$ Clinical Pharmacology, Institute of Infection and Immunity, St George's University of London, London, UK; ${ }^{3}$ St George's University of London, London, UK; ${ }^{4}$ Academic Respiratory Unit, University of Bristol, Bristol, UK

Correspondence: Emma H Baker Clinical Pharmacology, Institute of Infection and Immunity, Mail Point JIA, St George's University of London, Cranmer Terrace, London SWI7 ORE, UK Tel +44208725 5383

Email ebaker@sgul.ac.uk
Purpose: People with COPD have cognitive dysfunction, which is greater in those hospitalized for exacerbations than in stable outpatients. We tested the hypothesis that cognitive dysfunction at exacerbation is a disease-specific feature of COPD, rather than a nonspecific feature of hospitalization for acute illness, by comparing cognition between patients hospitalized for acute COPD exacerbations and those with worsening heart failure (HF).

Patients and methods: A total of 40 hospital inpatients were recruited, 20 patients with COPD exacerbations and 20 patients with congestive or left-sided HF. Exclusion criteria included previous stroke, known neurological disease, and marked alcohol excess. Participants completed the Montreal cognitive assessment (MoCA) and Hospital Anxiety and Depression Scale (HADS) and underwent spirometry and review of clinical records.

Results: Age (mean \pm SD, COPD $73 \pm 10$; HF $76 \pm 11$ years), acute illness severity (Acute Physiology and Chronic Health Evaluation [APACHE]-II, COPD 15.4 \pm 3.5 ; HF 15.9 \pm 3.0 ), comorbidities (Charlson index, COPD 1.3 \pm 1.9 ; HF 1.6 \pm 1.5 ), and educational background were similar between COPD and HF groups. MoCA total was significantly lower in COPD than in HF (COPD 20.6 \pm 5.6 ; HF 24.8 $\pm 3.5, P=0.007$ ); however, significance was lost after correction for age, sex, and pack year smoking history. When compared with HF patients, the COPD cohort performed worse on the following domains of the MoCA: visuospatial function (median [IQR], COPD 0 [1]; HF 2 [1], $P=0.003$ ), executive function (COPD 2 [1]; HF 3 [1], $P=0.035$ ), and attention (COPD 4 [3]; HF 6 [2], $P=0.020)$. Age $(P=0.012)$ and random glucose concentration $(P=0.041)$ were associated with cognitive function in whole group analysis, with pack year smoking history reaching borderline significance $(P=0.050)$.

Conclusion: Total MoCA score for COPD and HF indicated that both groups had mild cognitive impairment, although this was greater in people with COPD. Mechanisms underlying the observed cognitive dysfunction in COPD remain unclear but appear related to blood glucose concentrations and greater lifetime smoking load.

Keywords: Montreal cognitive assessment, cognition, comorbidities, systemic, smoking, hyperglycemia

\section{Introduction}

Cognitive dysfunction is common in people with COPD. In a recent systematic review, cognitive impairment was reported to affect one in three COPD patients. ${ }^{1}$ Impaired cognition in people with COPD is associated with reduced treatment adherence, impaired performance in daily activities, and increased mortality. For example, in an observational study of 157 patients referred for pulmonary rehabilitation, patients with cognitive impairment were twice as likely to fail to complete submit your manuscript Dovepress f http in $\square$ 
the program as those without. $^{2}$ In a systematic review of 13 studies assessing the impact of cognitive impairment on self-management in COPD, cognitive dysfunction was associated with an increased need for assistance in activities of daily living, reduced symptom recall, and increased errors in inhaler technique. ${ }^{3}$ Understanding what drives cognitive impairment in people with COPD is therefore important in order to identify factors that could be modified to improve health outcomes.

Mild cognitive impairment refers to a decline in cognition, which presents as the intermediary stage between normal cognitive aging and dementia, independent of educational level and not severe enough to interfere with daily activities. ${ }^{4}$ Longitudinal studies have reported that one-half of individuals who meet the criteria for mild cognitive impairment develop dementia within 3 years. ${ }^{5}$

While the mechanisms that underlie cognitive impairment in COPD are not completely understood and are likely to be multifactorial, COPD-specific factors have been proposed. ${ }^{6}$ These include hypoxemia, ${ }^{7}$ disease severity, ${ }^{8}$ reduced physical activity, ${ }^{9}$ and exacerbations. ${ }^{10,11}$ Hospitalization due to the exacerbations of COPD in particular appears to be a high risk for cognitive dysfunction. A study comparing 30 COPD patients hospitalized with exacerbations, 50 stable COPD patients, and 30 age-matched healthy controls found that $50 \%$ of hospitalized patients had impaired processing speed compared to $24 \%$ of stable COPD patients and 3\% of controls. ${ }^{11}$ Moreover, hospitalized patients deemed medically fit for discharge had significant deficits in cognitive function, which had not improved 3 months after discharge. ${ }^{11}$ In a 2 -year prospective cohort study of patients hospitalized with COPD exacerbations, baseline assessment of cognitive function was significantly worse in those who died during follow-up than in those who survived. ${ }^{12}$ What remains unclear is whether the cognitive dysfunction seen in hospitalized COPD patients is due to a COPD-specific effect or rather a nonspecific effect of acute illness and hospitalization itself.

The hypothesis underlying this study is that patients hospitalized due to COPD exacerbations are more likely to have cognitive impairment than those hospitalized with decompensation of other chronic illnesses, due to COPD-specific factors. To test this hypothesis, we compared the prevalence of cognitive impairment in hospitalized patients with COPD exacerbations and decompensated heart failure (HF) and investigated the relationship between cognitive impairment and clinical characteristics within disease groups.

\section{Methods}

\section{Design}

This was a prospective case-control study. All participants gave written informed consent. The study was approved by the West Midlands Research Ethics Committee (13/ WM/0434).

\section{Patient selection}

Patients admitted to hospital with the exacerbations of COPD were recruited and compared with patients hospitalized with decompensated HF. HF patients were selected as a control group for comparison with COPD patients because a similarly high prevalence of cognitive impairment has been reported in both diseases. ${ }^{1}$ They were also expected to be comparable in terms of age and comorbidities.

\section{Inclusion criteria}

COPD patients were identified on admission with a physician diagnosis of an acute exacerbation of COPD. Participants had symptoms of exacerbation (cough, sputum, and breathlessness), a significant smoking history ( $>10$ pack years), and obstructive spirometry (ratio of $\mathrm{FEV}_{1}$ to FVC <0.7).

HF patients were identified on admission with a physician diagnosis of decompensated HF. Participants had edema and/or dyspnea, a prior diagnosis of HF, evidence of reduced ejection fraction, and/or diastolic dysfunction on echocardiogram.

\section{Exclusion criteria}

Patients were excluded if they had combined COPD and $\mathrm{HF}$, asthma, pneumonia, past medical history of stroke, neurological disorder, psychiatric disorder, marked alcohol excess (male $>56 \mathrm{U} /$ week, female $>42 \mathrm{U} /$ week), or severe renal failure $(>500 \mu \mathrm{mol} / \mathrm{L}$ creatinine) (Figure 1).

\section{Clinical measures}

Two examiners performed the clinical exams to ensure coverage of the patients who came in as emergencies. Both examiners were trained to perform research techniques by the same clinical research coordinator. Both examiners assessed patients from both groups.

\section{Evaluation of comorbidities and airflow limitation}

Comorbidities were measured using the Charlson comorbidity index. ${ }^{13}$ Anxiety and depression were assessed using the Hospital Anxiety and Depression Scale (HADS, maximum score 42$).{ }^{14}$ The HADS is split into anxiety (21 points) 


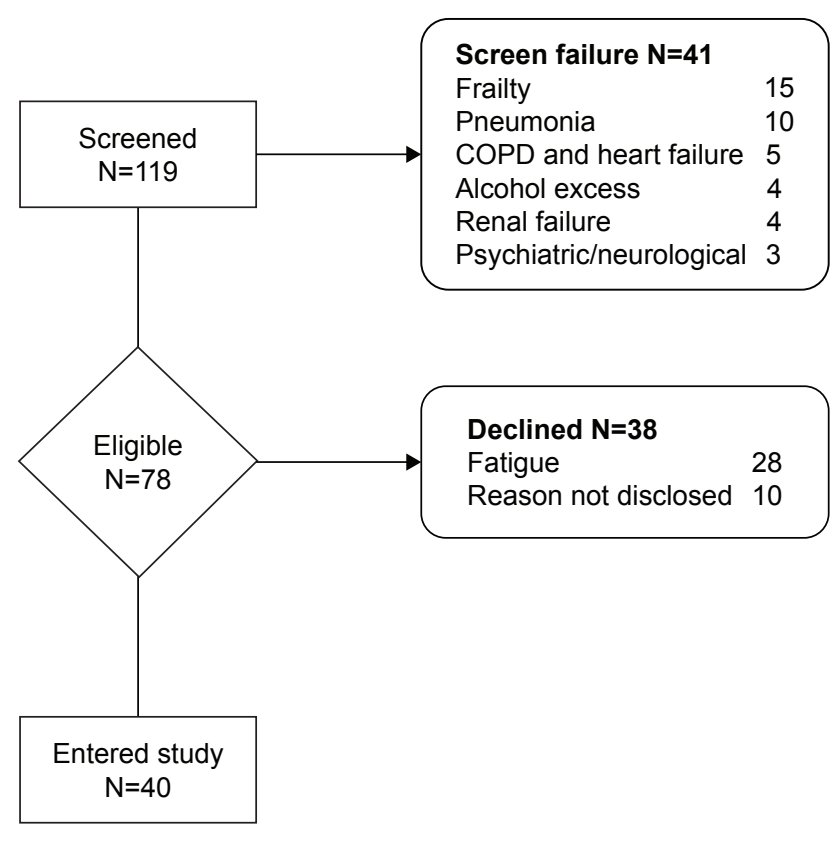

Figure I Flowchart of patient recruitment.

and depression (21 points) subscales, and the scores are categorized as follows: $0-7$ points, normal; $8-10$ points, mild; 11-14 points, moderate; and 15-21 points, severe. Illness severity was measured using the Acute Physiology and Chronic Health Evaluation (APACHE-II, maximum score 71). ${ }^{15}$ The burden of COPD symptoms was measured using the COPD assessment test (CAT). ${ }^{16}$ The CAT scores are characterized as follows: $0-10$ points, mild; $11-20$ points, moderate; $21-30$ points, severe; and $31-40$ points, very severe, clinical impact.

Airflow limitation was measured according to standardized spirometry guidelines ${ }^{17}$ using the EasyOneTM Worldspirometer device (NDD Medical Technologies). Absolute $\mathrm{FEV}_{1}, \mathrm{FVC}, \mathrm{FEV}_{1}: \mathrm{FVC}$, and percentage predicted measurements were obtained.

\section{Cognitive status}

The presence of delirium was assessed with the confusion assessment method (CAM) ${ }_{18}^{18}$ a bedside diagnostic tool that consists of nine operationalized criteria from the Diagnostic and Statistical Manual of Mental disorders (DSM-III-R). The Montreal cognitive assessment (MoCA) ${ }^{19}$ was used to assess the following cognitive domains: visuoconstructional skills, executive function, naming, delayed recall, attention, language, abstraction, and orientation. MoCA was scored out of a total of 30 points, and a cutoff of less than 26 was considered indicative of mild cognitive impairment.

\section{Statistical analyses}

Continuous data were described using mean $\pm \mathrm{SD}$, and categorical data were described as number (\%) participants. Normally distributed variables were compared using the independent $t$-test and non-normally distributed variables by Mann-Whitney $U$ tests. Categorical variables were compared using Chi-squared tests. Analysis of covariance (ANCOVA) was used to control for age, sex, and pack year smoking history, during the comparison of normally distributed continuous variables. Group-specific associations between MoCA and clinical measures were tested using Pearson's correlation and corrected for age and sex. Pack year smoking history was not corrected in the correlational analysis in order to investigate its effect. Where values were not normally distributed they were log-transformed before correlations were performed. Variables, which were significantly associated with MoCA, were further analyzed using ANCOVA. The ANCOVA model tested for the following main effects: dependent variable, MoCA total; fixed factors, group (COPD/ HF); covariates, age, sex, random glucose concentration, and pack year smoking history. The following interactions were also assessed - group by random glucose concentration and group by pack year smoking history. All statistical analyses were performed using IBM SPSS ${ }^{\circledR}$ (version 21.0).

\section{Results}

\section{Patient demographics}

A total of 20 COPD patients and 20 patients with HF were recruited. Demographics and clinical characteristics are compared in Table 1.

\section{COPD and HF patients have similar comorbidities and acute illness severity}

COPD patients and HF patients were well-matched for age, comorbidities (Charlson comorbidity index), and acute illness severity (APACHE-II score). As expected, COPD patients had worse airflow obstruction, greater smoking pack years, and more respiratory symptoms (CAT) and were more likely to be receiving bronchodilators (beta-2 agonists and anticholinergics) and either inhaled or systemic corticosteroids. Conversely, HF patients were more likely to be taking cardiac medications such as diuretics, angiotensinconverting enzyme (ACE) inhibitors, beta-blockers, and aldosterone antagonists. Interestingly, despite the differences in underlying diagnosis, both groups had elevated systemic inflammatory markers (C-reactive protein [CRP]). COPD patients had a higher circulating white blood count, 
Table I Clinical and demographic characteristics of participants

\begin{tabular}{|c|c|c|c|}
\hline & COPD $(n=20)$ & Heart failure $(n=20)$ & $P$-value \\
\hline Age (years) & $73 \pm 10$ & $76 \pm 11$ & 0.392 \\
\hline Sex, n (\%) (male) & $8(40 \%)$ & $14(70 \%)$ & 0.057 \\
\hline Charlson index & $1.3 \pm 1.9$ & $1.6 \pm 1.5$ & 0.314 \\
\hline APACHE-II score & $15.4 \pm 3.5$ & $15.9 \pm 3.0$ & 0.565 \\
\hline Smoking history (pack years) & $51.5 \pm 35.0$ & $\mid I . I \pm I 5.6$ & 0.001 \\
\hline Alcohol (units/week) & $4.5 \pm 7.1$ & $9.1 \pm 13.1$ & 0.265 \\
\hline Admission to assessment (days) & $3.1 \pm 3.4$ & $7.9 \pm 11.1$ & 0.149 \\
\hline $\mathrm{FEV}_{1}(\%$ predicted $)$ & $42 \pm 19$ & $53 \pm 16$ & 0.101 \\
\hline $\mathrm{FEV}_{1}$ :FVC (\%) & $56 \pm 14$ & $71 \pm 16$ & 0.024 \\
\hline Oxygen saturations (\%) & $93 \pm 4$ & $97 \pm 2$ & 0.001 \\
\hline HADS anxiety (maximum 21 points) & $9 \pm 6$ & $6 \pm 4$ & 0.047 \\
\hline HADS depression (maximum 21 points) & $10 \pm 4$ & $9 \pm 4$ & 0.216 \\
\hline C-reactive protein (mg/L) & $36.3 \pm 49.7$ & $31.3 \pm 31.0$ & 0.512 \\
\hline White blood cell count $\left(10^{9} / \mathrm{L}\right)$ & $13 \pm 6$ & $9 \pm 3$ & 0.003 \\
\hline Random glucose (mmol/L) & $8.2 \pm 2.8$ & $8.0 \pm 3.0$ & 0.683 \\
\hline Total number of drugs $(n)$ & $8.6 \pm 3.3$ & $9.9 \pm 3.2$ & 0.229 \\
\hline Drugs for COPD $(n)^{a}$ & $3.7 \pm 0.9$ & $0.6 \pm 1.1$ & $<0.001$ \\
\hline Drugs for heart failure $(n)^{b}$ & $0.6 \pm 0.6$ & $2.8 \pm 0.6$ & $<0.001$ \\
\hline Drugs affecting cognition $(n)^{c}$ & $0.8 \pm 1.2$ & $0.4 \pm 0.8$ & 0.162 \\
\hline CAT (maximum 40 points) & $27.8 \pm 6.4$ & $21.4 \pm 8.4$ & 0.010 \\
\hline
\end{tabular}

Notes: Bold values denote statistical significance. ${ }^{a}$ Beta-2 agonists, anticholinergics, and corticosteroids. ${ }^{b}$ Diuretics, angiotensin-converting enzyme inhibitors, beta-blockers, and aldosterone antagonists. 'Opioids, sedatives, antimuscarinics, and antidepressants.

Abbreviations: APACHE, Acute Physiology and Chronic Health Evaluation; CAT, COPD assessment test; HADS, Hospital Anxiety and Depression Scale.

which in the absence of differences in CRP is most likely due to systemic corticosteroid treatment. The groups were well-matched for other factors that might affect cognition, including alcohol consumption and sedative medication. COPD patients were significantly more anxious, but not more depressed, than patients with HF (Table 1). A total of 50\% of people with HF and only $10 \%$ of people with COPD had diabetes mellitus $(P=0.014)$. Random blood glucose did not differ between groups.

\section{Cognitive impairment is common in COPD patients even when delirium is infrequent}

A total of $15 \%$ of the patients in the COPD group showed evidence of delirium, compared to no patients in the HF group. The MoCA score was 4 points (95\% CI 1-7) lower in COPD patients than in people with $\mathrm{HF}$ (mean $\pm \mathrm{SD}$, COPD 20.6 \pm 5.6 ; HF 24.8 $\pm 3.5, P=0.007)$. Statistical significance was lost after controlling for age, sex, and smoking pack year history $(P=0.292)$. Using the MoCA score $<26$ points as suggestive of cognitive impairment, $90 \%$ of people in the COPD group had cognitive impairment compared to $55 \%$ of people in the HF group. Reviewing MoCA sub-domains,
COPD patients scored significantly lower in the cognitive domains of visuospatial function, executive function, and attention. Naming, language, abstraction, delayed recall, and orientation were not significantly different between the groups (Table 2).

\section{In COPD patients, poor cognitive} performance is associated with greater smoking history but not lung function or oxygen saturation

In the COPD group, MoCA score was inversely correlated with smoking pack year history (Table 3 ). There was no association between MoCA score and inflammatory markers (white blood cell count and CRP), hypoxemia (oxygen saturations), lung function ( $\mathrm{FEV}_{1} \%$ predicted), illness severity (APACHE-II, CAT), anxiety and depression (HADS), or random glucose concentration.

\section{In HF patients, poor cognitive} performance was associated with higher random blood glucose concentration

In people with HF, MoCA score was negatively correlated with random glucose concentration (Table 4). There was no 
Table 2 Cognitive assessment

\begin{tabular}{|l|l|l|l|}
\hline MoCA cognitive domain & COPD $(\mathbf{n}=\mathbf{2 0})$ & Heart failure $(\mathbf{n}=\mathbf{2 0})$ & P-value \\
\hline Total (maximum 30 points) & $20.6 \pm 5.6$ & $24.8 \pm 3.5$ & $\mathbf{0 . 0 0 7}$ \\
\hline Visuospatial function (maximum 2 points) & $0(\mathrm{I})$ & $2(\mathrm{I})$ & $\mathbf{0 . 0 0 3}$ \\
\hline Executive function (maximum 3 points) & $2(\mathrm{I})$ & $3(\mathrm{I})$ & $\mathbf{0 . 0 3 5}$ \\
\hline Naming (maximum 3 points) & $3(\mathrm{I})$ & $3(\mathrm{I})$ & 0.947 \\
\hline Attention (maximum 6 points) & $4(3)$ & $6(2)$ & $\mathbf{0 . 0 2 0}$ \\
\hline Language (maximum 3 points) & $2(2)$ & $3(\mathrm{I})$ & 0.068 \\
\hline Abstraction (maximum 2 points) & $\mathrm{I}(2)$ & $2(\mathrm{I})$ & 0.165 \\
\hline Delayed recall (maximum 5 points) & $3(4)$ & $3(2)$ & 0.718 \\
\hline Orientation (maximum 6 points) & $6(0)$ & $6(0)$ & 0.738 \\
\hline
\end{tabular}

Notes: Gaussian data are presented as mean \pm SD and non-Gaussian data are presented as median (IQR). Bold values denote statistical significance where $P<0.05$. Abbreviation: MoCA, Montreal cognitive assessment.

association between MoCA score and inflammatory markers (white blood cell count and CRP), hypoxemia (oxygen saturations), lung function ( $\mathrm{FEV}_{1} \%$ predicted), smoking pack years, illness severity (APACHE-II, CAT), or anxiety and depression (HADS).

\section{In the whole group, age, random glucose concentration, and pack year smoking history were independently associated with cognitive function}

The relationship between MoCA and underlying diagnosis (COPD or HF) was explored using an ANCOVA (Table 5). Group allocation, age, and sex were included in the model, along with factors that individually correlated with the MoCA in COPD and HF (smoking pack year history and random glucose concentration, respectively, Tables 3 and 4). In this model, diagnosis was not a significant determinant of cognitive function. However, age $(P=0.012)$, random glucose

Table 3 Correlations with MoCA in COPD

\begin{tabular}{|l|l|l|}
\hline & $\boldsymbol{R}$ value & $\boldsymbol{P}$-value \\
\hline APACHE-II score* & 0.352 & 0.152 \\
\hline Smoking history (pack years)* & -0.536 & $\mathbf{0 . 0 2 2}$ \\
\hline FEV $_{\text {(\% predicted) }}$ (\%) & -0.098 & 0.740 \\
\hline Oxygen saturations (\%) & -0.319 & 0.197 \\
\hline Random glucose (mmol/L)* & -0.447 & 0.145 \\
\hline White blood cell count (I0\%/L) & -0.180 & 0.476 \\
\hline CRP (mg/L)* & -0.447 & 0.081 \\
\hline CAT & -0.277 & 0.266 \\
\hline HADS total & -0.443 & 0.065 \\
\hline
\end{tabular}

Notes: Bold values denote statistical significance. *Non-Gaussian variables were log transformed for parametric analysis. Data presented are corrected for age and sex. Abbreviations: APACHE, Acute Physiology and Chronic Health Evaluation; CAT, COPD assessment test; CRP, C-reactive protein; HADS, Hospital Anxiety and Depression Scale; MoCA, Montreal cognitive assessment. concentration $(P=0.041)$, and pack year smoking history ( $P=0.050$ - borderline statistical significance) were significantly associated with cognitive function.

\section{Discussion}

The aim of this study was to determine if cognitive impairment was more prevalent in people hospitalized with COPD exacerbations than in people hospitalized due to decompensated HF. We found that patients with an acute exacerbation of COPD on average scored 4 points worse on the MoCA and were significantly more likely to have cognitive impairment, defined as MoCA $<26$, than those with decompensated HF. Statistical differences in cognitive function between groups did not survive adjustment for age, sex, and pack year smoking history. ANCOVA in the whole group found that age, random glucose concentration, and pack year smoking history, but not underlying diagnosis (COPD or HF), were independent determinants of cognitive function.

Table 4 Correlations with MoCA in heart failure

\begin{tabular}{|l|l|l|}
\hline & $\boldsymbol{R}$ value & $\boldsymbol{P}$-value \\
\hline APACHE-II score* & -0.105 & 0.679 \\
\hline Smoking history (pack years)* & -0.014 & 0.974 \\
\hline FEV $_{\text {, } \% \text { predicted) }}$ & -0.025 & 0.948 \\
\hline Oxygen saturations (\%) & -0.188 & 0.456 \\
\hline Random glucose (mmol/L)* & -0.676 & $\mathbf{0 . 0 1} \mathbf{~}$ \\
\hline White blood cell count (I0\%/L) & -0.348 & 0.158 \\
\hline CRP (mg/L)* & -0.299 & 0.229 \\
\hline CAT & -0.189 & 0.452 \\
\hline HADS total & -0.394 & 0.105 \\
\hline
\end{tabular}

Notes: Bold values denote statistical significance. *Non-Gaussian variables were log transformed for parametric analysis. Data presented are corrected for age and sex. Abbreviations: APACHE, Acute Physiology and Chronic Health Evaluation; CAT, COPD assessment test; CRP, C-reactive protein; HADS, Hospital Anxiety and Depression Scale; MoCA, Montreal cognitive assessment. 
Table 5 ANCOVA model of relationships with MoCA

\begin{tabular}{|l|l|l|l|}
\hline Variables & $\mathbf{F}(\mathbf{I}, \mathbf{I}$ 4) & P-value & $\begin{array}{l}\text { Partial Eta } \\
\text { squared }\end{array}$ \\
\hline Group (COPD/HF) & 0.235 & 0.636 & 0.016 \\
\hline Age & 8.406 & $\mathbf{0 . 0 1 2}$ & 0.375 \\
\hline Sex & 1.648 & 0.220 & 0.105 \\
\hline $\begin{array}{l}\text { Random glucose concentration } \\
\text { (mmol/L) }\end{array}$ & 5.079 & $\mathbf{0 . 0 4 I}$ & 0.266 \\
\hline Smoking history (pack years)* & 4.585 & 0.050 & 0.247 \\
\hline $\begin{array}{l}\text { Group by random glucose } \\
\text { concentration interaction }\end{array}$ & 1.671 & 0.217 & 0.107 \\
\hline $\begin{array}{l}\text { Group by smoking history } \\
\text { (pack years) interaction }\end{array}$ & 0.884 & 0.363 & 0.059 \\
\hline
\end{tabular}

Notes: Bold values denote statistical significance. ${ }^{*}$ Non-Gaussian variables were log transformed for parametric analysis.

Abbreviations: ANCOVA, analysis of covariance; HF, heart failure; MoCA, Montreal cognitive assessment.

Our findings of significant cognitive impairment in COPD patients hospitalized with exacerbations are consistent with other studies. Dodd et $\mathrm{al}^{11}$ reported that people hospitalized for COPD have greater cognitive impairment than stable outpatients with COPD and age-matched controls. That study is not directly comparable to ours as hospitalized patients in the Dodd study were at the point of discharge. López-Torres et $\mathrm{al}^{20}$ reported a mean MoCA total score of $19.28 \pm 2.08$ points in 48 patients hospitalized for acute exacerbation of COPD at admission, which is similar to the MoCA total of COPD patients in our study at $20.6 \pm 5.6$ points. Furthermore, consistent with our work, visuospatial function, executive function, and attentional deficits have previously been reported in COPD. ${ }^{6,21}$ Our study extends the findings of previous investigations in that we show that cognitive impairment in hospitalized COPD patients is greater than that in a hospitalized comparator group with decompensated HF.

We explored potential reasons underlying differences in cognition between hospitalized patients with COPD exacerbations or decompensated HF. Pack year smoking history differed markedly between the groups and was associated with cognitive dysfunction in COPD patients, independent of age and sex. In COPD, smoking load is significantly associated with more severe lung disease $\mathrm{e}^{22,23}$ and increased risk of hospitalization. ${ }^{24}$ Smoking is also a well-recognized cause of vascular disease, ${ }^{25}$ which can impair cerebral perfusion, altering cognition. ${ }^{26}$ Moreover, cigarette smoke is thought to contain particulates which have a direct neurotoxic effect. ${ }^{27}$ Greater smoking history could therefore contribute both to an increased risk of hospitalization and to the development of cognitive impairment, explaining the association. Smoking history of $>10$ pack years was an inclusion criterion for the COPD group and not for the HF group, who comprised people with HF from diverse etiology. The relatively light smoking load in those with HF could be considered less deleterious to cognitive function, which could explain why correcting for its effect removed the significant difference in MoCA total between the groups, despite patients with HF being older. ANCOVA showed that smoking pack year history marginally missed significance as a predictor of cognitive impairment, possibly due to a lack of statistical power and that this was not a group dependent effect. This suggests that cognitive impairment is not COPD specific but a smoking-specific effect.

Random glucose concentration was inversely correlated with MoCA in HF but not in COPD patients. In HF patients, elevated random glucose concentration could merely have been a marker for underlying diabetes, which affected $50 \%$ patients, and is independently associated with cognitive impairment. ${ }^{28}$ Mean random glucose concentration was the same in COPD patients as in HF patients, despite only $10 \%$ COPD patients having diagnosed diabetes mellitus. Elevated blood glucose is common during COPD exacerbations, ${ }^{29}$ at least in part due to corticosteroid therapy. ${ }^{30}$ Despite there being no significant difference in random glucose concentrations between groups, the ANCOVA revealed that random glucose concentration predicted cognitive function and that this was not diagnosis dependent. This is consistent with a larger study by Crane et al who reported that hyperglycemia may be a risk factor for dementia. ${ }^{31}$ Previous studies have proposed that "overspill" of inflammation from the lungs of COPD patients into the systemic circulation ${ }^{32}$ may promote a neuroinflammatory response. ${ }^{33}$ This could be particularly marked during exacerbations and drive cognitive impairment. In the present study, lack of differences in systemic inflammation (CRP) between groups and lack of associations of inflammatory markers (CRP, white cell count) with cognitive function indicated that cognitive impairment in this hospitalized COPD group was not explained by inflammation. White cell count did differ between groups, but in the absence of a difference in serum CRP, this is most likely explained by corticosteroid treatment for COPD exacerbation. Corticosteroids can have neuropsychiatric side effects. However, we have previously shown that the more marked cognitive impairment seen in hospitalized COPD patients does not resolve with recovery and cessation of corticosteroids. ${ }^{11}$ Several studies have reported a relationship between hypoxia and cognitive dysfunction in COPD. ${ }^{7,34,35}$ However, these studies are limited by variation in the definition of hypoxemia between studies, correlations are often weak, and most evidence relates to non-hospitalized COPD patients. ${ }^{6}$ Although in our study, oxygen saturations 
were lower in COPD than HF patients, there was no association between oxygen saturations and cognitive impairment.

\section{Limitations}

Studies in hospital inpatients with acute illness are difficult to conduct because recruitment depends on unpredictable hospital admissions and patients can be too unwell to participate when ill enough to be eligible. In this context, we were able to recruit 20 patients in each group. The small size of this study means that our results need to be interpreted with caution as there is a risk of type 2 error due to insufficient power. We selected people with HF as "controls" with a chronic disease other than COPD prone to hospitalization for exacerbations. COPD and HF affect people at similar ages and are associated with a similar background prevalence of cognitive impairment. ${ }^{1}$ Despite this, there were other significant differences between groups, particularly in smoking and diabetes. Matching for these characteristics would have been desirable, although difficult.

Premorbid cognition was not measured in this study, which is known to be a major determinant of cognitive function in terms of brain reserve, ${ }^{36}$ so it is unclear how this might have influenced our analysis.

Cognitive impairment was measured using the MoCA. As a brief screening instrument, the MoCA has clinical utility as a general measure of cognitive impairment. Although we have analyzed the subdomains of the MoCA, which indicate that COPD patients have impairments in visuospatial function, executive function, and attention, an in-depth battery of neuropsychological tests would be required to assess this fully.

Associations between the MoCA and clinical characteristics gave some clues as to the mechanisms underlying cognitive impairment in hospitalized COPD patients. However, as this is a cross-sectional study it is not possible to attribute direction of causation.

Finally, as two examiners performed the clinical exams, inter-rater variability should have been calculated to determine the consistency and reliability of the scoring. Despite this, both examiners assessed patients from both patient groups so there was no opportunity for systematic bias to occur.

Cognitive impairment has a significant clinical impact for COPD patients. As patients hospitalized with COPD exacerbations appear particularly vulnerable to cognitive impairment, they may be the group who would benefit most from targeted interventions. Our study found that COPD patients performed significantly worse on the visuospatial, executive function and attention domains of the MoCA compared to HF patients. Lezak et $\mathrm{al}^{37}$ described executive function as the ability to formulate goals, plan achievement, and perform behaviors effectively. Impairment in this area can result in inability to utilize and adhere to medication adequately as well as inability to perform other aspects of personal management. Visuospatial function involves the perception of orientation, spatial location, direction, and distance. Impairments in this area can lead to the inability to plan a route or leaving home and getting lost. Attention requires appropriate allocation of processing resources; therefore, dysfunction in this area would result in difficulty concentrating on tasks. ${ }^{38}$ Raising awareness of the existence of cognitive impairment, using a reliable tool, facilitated with support groups and health care contacts, may be the route to explore to ensure improved adherence to therapy and perhaps to reduce hospitalizations.

\section{Conclusion}

COPD patients hospitalized for exacerbations have marked cognitive impairment, which is not explained by hospitalization. Cognitive impairment is associated with pack year smoking history, rather than systemic inflammation or hypoxia, indicating that smoking could be an important underlying mechanism. In hospitalized patients with either COPD or HF, random blood glucose was associated with cognitive impairment, indicating a potential contributing role for diabetes. Awareness of the prevalence of cognitive impairment in hospitalized patients with COPD has importance for treatment adherence and discharge planning to reduce readmissions.

\section{Disclosure}

JWD reports a British Lung Foundation research grant, personal fees from Boerhinger Ingelheim, and non-financial support from NAPP Pharmaceutical, and DRB reports a National Institute for Health Research academic grant, outside the submitted work. PWJ is also employed as a Global Medical Expert by GlaxoSmithKline. The other authors report no conflicts of interest in this work.

\section{References}

1. Yohannes AM, Chen W, Moga AM, Leroi I, Connolly MJ. Cognitive impairment in chronic obstructive pulmonary disease and chronic heart failure: a systematic review and meta-analysis of observational studies. J Am Med Dir Assoc. 2017;18(5):451.e1-4551.e11.

2. Cleutjens FAHM, Spruit MA, Ponds RWHM, et al. The impact of cognitive impairment on efficacy of pulmonary rehabilitation in patients with COPD. J Am Med Dir Assoc. 2017;18(5):420-426.

3. Baird C, Lovell J, Johnson M, Shiell K, Ibrahim JE. The impact of cognitive impairment on self-management in chronic obstructive pulmonary disease: a systematic review. Respir Med. 2017;129:130-139. 
4. Petersen RC, Smith GE, Waring SC, Ivnik RJ, Tangalos EG, Kokmen E. Mild cognitive impairment: clinical characterization and outcome. Arch Neurol. 1999;56(3):303-308.

5. Gauthier S, Reisberg B, Zaudig M, et al; International Psychogeriatric Association Expert Conference on mild cognitive impairment. Mild cognitive impairment. Lancet. 2006;367(9518):1262-1270.

6. Dodd JW, Getov SV, Jones PW. Cognitive function in COPD. Eur Respir J. 2010;35(4):913-922.

7. Grant I, Heaton RK, McSweeny AJ, Adams KM, Timms RM. Neuropsychologic findings in hypoxemic chronic obstructive pulmonary disease. Arch Intern Med. 1982;142(8):1470-1476.

8. Klein M, Gauggel S, Sachs G, Pohl W. Impact of chronic obstructive pulmonary disease (COPD) on attention functions. Respir Med. 2010; 104(1):52-60.

9. Sink KM, Espeland MA, Castro CM, et al; LIFE Study Investigators. Effect of a 24-month physical activity intervention vs health education on cognitive outcomes in sedentary older adults: the life randomized trial. JAMA. 2015;314(8):781-790.

10. Tulek B, Atalay NB, Yildirim G, Kanat F, Süerdem M. Cognitive function in chronic obstructive pulmonary disease: relationship to global initiative for chronic obstructive lung disease 2011 categories. Respirology. 2014;19(6):873-880.

11. Dodd JW, Charlton RA, van den Broek MD, Jones PW. Cognitive dysfunction in patients hospitalized with acute exacerbation of COPD. Chest. 2013;144(1):119-127.

12. Almagro P, Calbo E, Ochoa de Echagüen A, et al. Mortality after hospitalization for COPD. Chest. 2002;121(5):1441-1448.

13. Charlson ME, Pompei P, Ales KL, MacKenzie CR. A new method of classifying prognostic comorbidity in longitudinal studies: development and validation. J Chronic Dis. 1987;40(5):373-383.

14. Zigmond AS, Snaith RP. The hospital anxiety and depression scale. Acta Psychiatr Scand. 1983;67(6):361-370.

15. Knaus WA, Zimmerman JE, Wagner DP, Draper EA, Lawrence DE. APACHE-acute physiology and chronic health evaluation: a physiologically based classification system. Crit Care Med. 1981;9(8):591-597.

16. Jones PW, Harding G, Berry P, Wiklund I, Chen WH, Kline Leidy N. Development and first validation of the COPD Assessment Test. Eur Respir J. 2009;34(3):648-654.

17. Moore VC. Spirometry: step by step. Breathe. 2012;8(3):232-240.

18. Inouye SK, van Dyck CH, Alessi CA, Balkin S, Siegal AP, Horwitz RI. Clarifying confusion: the confusion assessment method. A new method for detection of delirium. Ann Intern Med. 1990;113(12):941-948.

19. Nasreddine ZS, Phillips NA, Bédirian V, et al. The Montreal Cognitive Assessment, MoCA: a brief screening tool for mild cognitive impairment. J Am Geriatr Soc. 2005;53(4):695-699.

20. López-Torres I, Valenza MC, Torres-Sánchez I, Cabrera-Martos I, Rodriguez-Torres J, Moreno-Ramírez MP. Changes in cognitive status in COPD patients across clinical stages. COPD. 2016;13(3):327-332.

21. Antonelli-Incalzi R, Corsonello A, Trojano L, et al. Screening of cognitive impairment in chronic obstructive pulmonary disease. Dement Geriatr Cogn Disord. 2007;23(4):264-270.
22. Riesco JA, Alcázar B, Trigueros JA, Campuzano A, Pérez J, Lorenzo JL. Active smoking and COPD phenotype: distribution and impact on prognostic factors. Int J Chron Obstruct Pulmon Dis. 2017; 12:1989-1999.

23. Zhang J, Lin XF, Bai CX. Comparison of clinical features between nonsmokers with COPD and smokers with COPD: a retrospective observational study. Int J Chron Obstruct Pulmon Dis. 2014;9:57-63.

24. Montserrat-Capdevila J, Godoy P, Marsal JR, Barbé F. Predictive model of hospital admission for COPD exacerbation. Respir Care. 2015; 60(9):1288-1294.

25. Durazzo TC, Meyerhoff DJ, Nixon SJ. Chronic cigarette smoking: implications for neurocognition and brain neurobiology. Int J Environ Res Public Health. 2010;7(10):3760-3791.

26. Gupta N, Simpkins AN, Hitomi E, Dias C, Leigh R, NIH natural history of stroke investigators. White matter hyperintensity-associated bloodbrain barrier disruption and vascular risk factors. J Stroke Cerebrovasc Dis. 2018;27(2):466-471.

27. Swan GE, Lessov-Schlaggar CN. The effects of tobacco smoke and nicotine on cognition and the brain. Neuropsychol Rev. 2007;17(3): 259-273.

28. Zilliox LA, Chadrasekaran K, Kwan JY, Russell JW. Diabetes and Cognitive Impairment. Curr Diab Rep. 2016;16(9):87.

29. Baker EH, Janaway CH, Philips BJ, et al. Hyperglycaemia is associated with poor outcomes in patients admitted to hospital with acute exacerbations of chronic obstructive pulmonary disease. Thorax. 2006; 61(4):284-289

30. Walters JA, Walters EH, Wood-Baker R. Oral corticosteroids for stable chronic obstructive pulmonary disease. Cochrane Database Syst Rev. 2005;(3):CD005374.

31. Crane PK, Walker R, Hubbard RA, et al. Glucose levels and risk of dementia. N Engl J Med. 2013;369(6):540-548.

32. Decramer M, Rennard S, Troosters T, et al. COPD as a lung disease with systemic consequences - clinical impact, mechanisms, and potential for early intervention. COPD. 2008;5(4):235-256.

33. Barnes PJ. Chronic obstructive pulmonary disease: effects beyond the lungs. PLoS Med. 2010;7(3):e1000220.

34. Huppert FA. Memory impairment associated with chronic hypoxia. Thorax. 1982;37(11):858-860.

35. Stuss DT, Peterkin I, Guzman DA, Guzman C, Troyer AK. Chronic obstructive pulmonary disease: effects of hypoxia on neurological and neuropsychological measures. J Clin Exp Neuropsychol. 1997;19(4):515-524.

36. Livingston G, Sommerlad A, Orgeta V, et al. Dementia prevention, intervention, and care. Lancet. 2017;390(10113):2673-2734.

37. Lezak MD, Howieson DB, Loring DW, Hannay HJ, Fischer JS. Neuropsychological Assessment. 4th ed. New York: NY, US: Oxford University Press; 2004.

38. American Psychiatric Association. Diagnostic and Statistical Manual of Mental Disorders. 5th ed. Arlington, VA: American Psychiatric Association; 2013.
Clinical Interventions in Aging

\section{Publish your work in this journal}

Clinical Interventions in Aging is an international, peer-reviewed journal focusing on evidence-based reports on the value or lack thereof of treatments intended to prevent or delay the onset of maladaptive correlates of aging in human beings. This journal is indexed on PubMed Central, MedLine,
Dovepress

CAS, Scopus and the Elsevier Bibliographic databases. The manuscript management system is completely online and includes a very quick and fair peer-review system, which is all easy to use. Visit http://www.dovepress. com/testimonials.php to read real quotes from published authors. 